\title{
FITTING HELICAL SNAKE AND ROTATOR FIELD STRENGTH MEASUREMENTS IN RHIC
}

\author{
V. Ranjbar, A. U. Luccio,W. W. MacKay, N. Tsoupas, BNL, Upton, NY \\ S.Y.Lee, Indiana University, Bloomington, IN
}

\begin{abstract}
We examined recent multi-pole measurements for the helical snakes and rotators in RHIC to generate a full field map. Since multi-pole measurements yield real field values for $B_{\rho}$ field components we developed a unique technique to evaluate the full fields using a traditional finite element analysis software [1]. From these measurements we employed SNIG [2] to generate orbit and Spin plots. From orbit values we generated a transfer matrix for the first snake.
\end{abstract}

\section{FIELD ANALYSIS PROBLEM}

Much work has been done on predicting the field structure of the newly installed helical snakes and rotators in RHIC. Analytical[3] [4] [5] [7] and Numerical [6] work has been conducted to generate an appropriate model for these elements. But up to now actual field measurements taken from the real magnets have not been analyzed. A comparison of the actual field values with current analytical models proved problematic in terms of fitting. In addition to the problem of modeling the end effects and accounting for the quadrupole fields, we found a general longitudinal dependence for the multi-poles in the helical basis. Since this longitudinal dependence could not be accounted for in a neat analytical solution and required resorting to a series solution expansion we decided that using an available finite element analysis program would be a more efficient method to generate a solution.

However current finite element analysis programs are designed to solve Laplace's equation for cases with a scalar potential boundary condition. Since we possessed multipole data appropriate for the generation $B_{\rho}$ field components along a $3.1 \mathrm{~cm}$ radius, we needed to develop a magnetic scalar potential along a cylindrical surface to use the software to solve the interior field problem. Considering that the $B_{\rho}$ component must satisfy Laplace's equation separately:

$$
\nabla^{2} B_{\rho}=0
$$

We can use TOSCA [1] to solve this version of Laplace's equation thus giving $B_{\rho}$ everywhere interior to our boundary conditions $\left(\rho_{0}=3.1 \mathrm{~cm}\right)$. Using this $B_{\rho}$ we can evaluate the real magnetic scalar potential $\Phi_{M}$ using:

$$
\Phi\left(\rho_{0}\right)_{M}=\int_{0}^{\rho_{0}} B_{\rho} d \rho+\Phi(0)_{M}
$$

Finally using our derived values for $\Phi_{M}$ we can again use TOSCA [1] as it was intended, generating a useable full field model contained in the OPERA-TOSCA [1] operating environment.
Since we are equipped to solve for fields internal to our known boundary conditions, we are restricted to considering transverse particle motion of $\rho<3.1 \mathrm{~cm}$. Given a beam pipe with an internal radius of $4.5 \mathrm{~cm}$ it would be better if we could account for displacements up to at least $4.1 \mathrm{~cm}$. To accomplish this we simply linearly extrapolated the straight magnetic field formula for $B_{\rho}$

$$
\begin{aligned}
B_{\rho}=B_{0} \sum_{n=0}^{\infty}\left(\frac{\rho}{\rho_{0}}\right)^{n}\left[a_{n} \cos ((n\right. & +1) \theta) \\
& \left.+b_{n} \sin ((n+1) \theta)\right]
\end{aligned}
$$

by simply using existing multi-pole values and evaluating $B_{\rho}$ at a $4.1 \mathrm{~cm}$ radius.

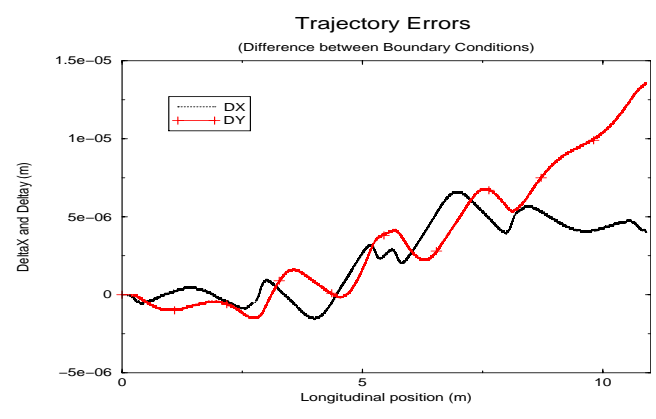

Figure 1: Difference in $\mathrm{x}$ and $\mathrm{y}$ trajectories for Fields evaluated with boundary conditions at $3.1 \mathrm{~cm}$ and $4.1 \mathrm{c} . \mathrm{m}$.

From Fig 1 it seems clear that using a $4.1 \mathrm{~cm}$ radius for the boundary conditions will give reasonable orbit results.

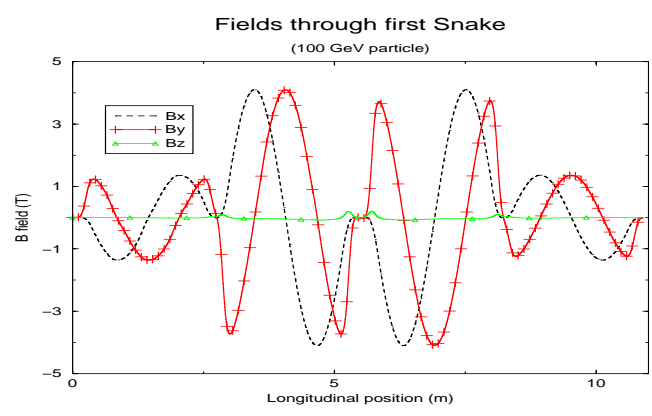

Figure 2: $B_{x}, B_{y}$ and $B_{z}$ fields evaluated along $100 \mathrm{GeV}$ particle path.

\section{ORBITAL TRAJECTORY THROUGH FIRST SNAKE}

Using SNIG [2] we track $100 \mathrm{GeV}$ proton through the Field map of a single snake. Results were consistent with 


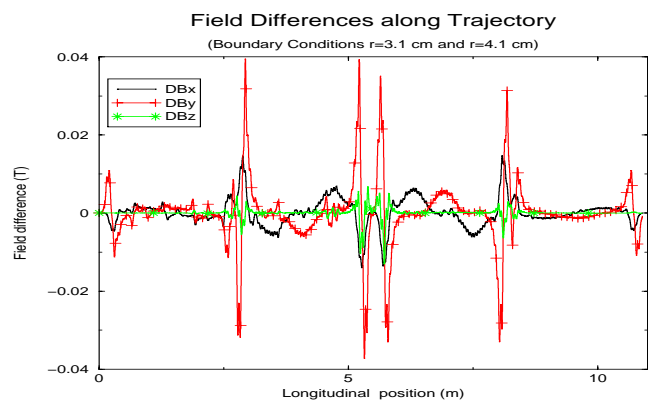

Figure 3: delta $B_{x}$, delta $B_{y}$ and delta $B_{z}$ fields evaluated along $100 \mathrm{GeV}$ particle path comparing $\rho=3.1 \mathrm{~cm}$ to a $4.1 \mathrm{~cm}$

previous predictions, however construction errors lead to an exiting orbit step of $-0.289 \mathrm{~mm}$ and $-0.489 \mathrm{~mm}$ in the $x$ and $y$ direction respectively. In addition the particle picked up a bend of $-0.46 \mathrm{mrad}$ and $-1.21 \mathrm{mrad}$ in $x^{\prime}$ and $y^{\prime}$.

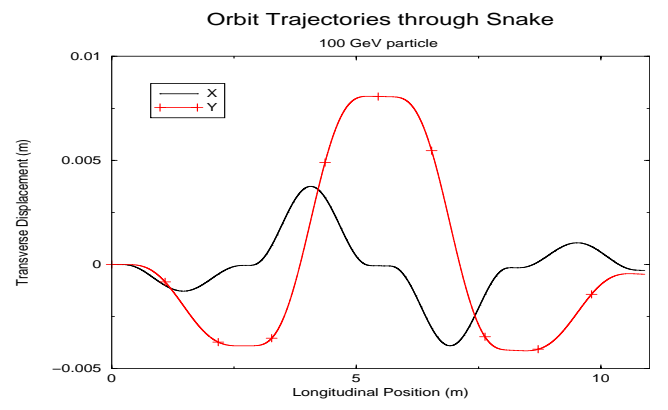

Figure 4: $\mathrm{X}$ and $\mathrm{Y}$ trajectories through full snake.

A quick evaluation of the orbit paths for particles entering close to the axis and small transverse momentum yielded the following transfer matrix:

$$
\begin{gathered}
M= \\
\left(\begin{array}{cccc}
0.9964174 & 10.980413 & 0.0014789 & 0.0011432 \\
-0.000656 & 0.9985205 & 0.0002366 & 0.0000774 \\
0.0001979 & 0.0126185 & 0.9883464 & 10.924713 \\
0.0002217 & 0.0038101 & -0.002115 & 0.9880669
\end{array}\right)
\end{gathered}
$$

which yields a $|M|=1.002$ from this matrix it is clear that the snakes does induce some coupling. The contribution of this coupling to spin resonances has been evaluated [9] showing a strength on the order of coupling caused by the operation of the solenodial field in both PHENIX and STAR detectors. Checking for simplecticity gives:

$$
\left(\begin{array}{cccc}
M^{T} \cdot S \cdot M= \\
0 & 1.002 & 0.000017 & -0.0021 \\
-1.002 & 0 & -0.0027 & -0.029 \\
-0.000017 & 0.0027 & 0 & 1 \\
0.0021 & 0.029 & -1 & 0
\end{array}\right)
$$

Clearly this is inadequate for tracking purposes and more work must still be dones to improve the simplecticity of the snake matrix. As well a more robust technique needs to be employed to calculate the transfer maps up to second order similar to [8].

\section{SPIN TRAJECTORY}

Considering the behavior of spin through the snake we found our results matched fairly well with previous predictions. With the outer two magnets set at 102 Amps and the inner two at 329 amps the particle achieved a complete spin flip.

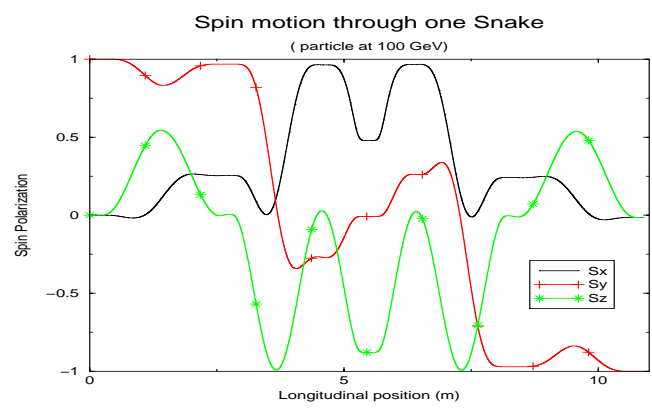

Figure 5: Sx Sy Sz Spin trajectories through full snake. Starting off with $S y=1$ polarization

Work needs still to done in evaluating the fields for the rest of the snakes and rotators in RHIC and running orbital and spin tracking under these fields.

\section{REFERENCES}

[1] TOSCA and OPERA commercial software.

[2] A.U.Luccio, Trends in Collider Spin Physics pp 244 World Scientific (1997)

[3] E.D. Courant, Orbit Matrices for Helical Snakes AGS/RHIC/Spin Note, BNL No. 4 (1996)

[4] E.D. Courant, Hybrid Helical Snakes and Rotators for RHIC, AGS/RHIC/Spin Note, BNL No. 10 (1996)

[5] M.J.Syphers, Closed Orbit Errors from Helical Dipole Magnets, AGS/RHIC/Spin Note, BNL No. 16 (1996)

[6] K.Hatanaka, T.Katayama,T.Tominaka, Maxwellian Field Expansion of Helical Magnet, IEEE 3416 (1998).

[7] W.Fischer, M. Okamura, Parameterization and measurements of Helical Magnetic Fields, IEEE 3341 (1998).

[8] A.U.Luccio, Field Map Generated Matrices for Spin Tracking, RIKEN-AF-NP-235 (1996)

[9] V.Ranjbar,S.Y.Lee,W.W.MacKay,M.Bai,E.Courant, Mapping out the full spin Resonance structure of RHIC, PAC2001 paper to be submitted 